\title{
Guanine Radicals Induced in DNA by Low-Energy
}

\section{Photoionization}

Evangelos Balanikas, ${ }^{1}$ Akos Banyasz, ${ }^{2}$ Thierry Douki,${ }^{3}$ Gérard Baldacchino ${ }^{1}$ and Dimitra

Markovitsi $*^{1}$

1. Université Paris-Saclay, CEA, CNRS, LIDYL, F-91191 Gif-sur-Yvette, France

2. Univ Lyon, ENS de Lyon, CNRS UMR 5182, Université Claude Bernard Lyon 1, Laboratoire

de Chimie, F-69342 Lyon, France

3. Univ. Grenoble Alpes, CEA, CNRS, IRIG, SyMMES, F-38000 Grenoble France 
CONSPECTUS. Guanine $(\mathbf{G})$ radicals are precursors to DNA oxidative damage, correlated with carcinogenesis and aging. During the past few years, we demonstrated clearly an intriguing effect: G radicals can be generated upon direct absorption of UV radiation with energy significantly lower than the $\mathbf{G}$ ionization potential. Using nanosecond transient absorption spectroscopy, we studied the primary species, ejected electrons and guanine radicals, which result from photoionization of various DNA systems in aqueous solution.

The DNA propensity to undergo electron detachment at low photon energies greatly depends on its secondary structure. Non-detected for monomers or unstacked oligomers, it may be one order of magnitude higher for G-quadruplexes than for duplexes. The experimental results point toward non-vertical processes, associated with the relaxation of electronic excited states. Theoretical studies are required to validate the mechanism and determine the factors that enter in play. In any case, such a mechanism, which may be operative over a broad excitation wavelength range, explains the occurrence of oxidative damage observed upon UVB and UVA irradiation.

Quantification of $\mathbf{G}$ radical populations and their time evolution questioned some widespread views. It appeared that $\mathbf{G}$ radicals may be generated with the same probability as pyrimidine dimers, which are considered to be the major lesions induced upon absorption of low-energy UV radiation by DNA. As the most important part of the initially created radical cations undergoes deprotonation, the vast majority of the final reaction products is expected to stem from longlived deprotonated radicals. Consequently, the widely used oxidation marker 8-oxodG is not representative of the extent of oxidative damage when this is triggered by generation of radical cations. 
Beyond the biological consequences, photo-generation of electron holes in G-quadruplexes may inspire applications in nano-electronics; although four-stranded structures are currently studied as molecular wires, their behavior as photoconductors has not been explored so far.

In the present Account, after highlighting some key experimental issues, we first describe the photo-ionization process and, then, we focus on radicals. We use as show-cases new results obtained for genomic DNA and Oxytricha G-quadruplexes. Generation and reaction dynamics of $\mathbf{G}$ radicals in these systems provide a representative picture of the phenomena reported previously for duplexes and G-quadruplexes, respectively.

CONSPECTUS Figure

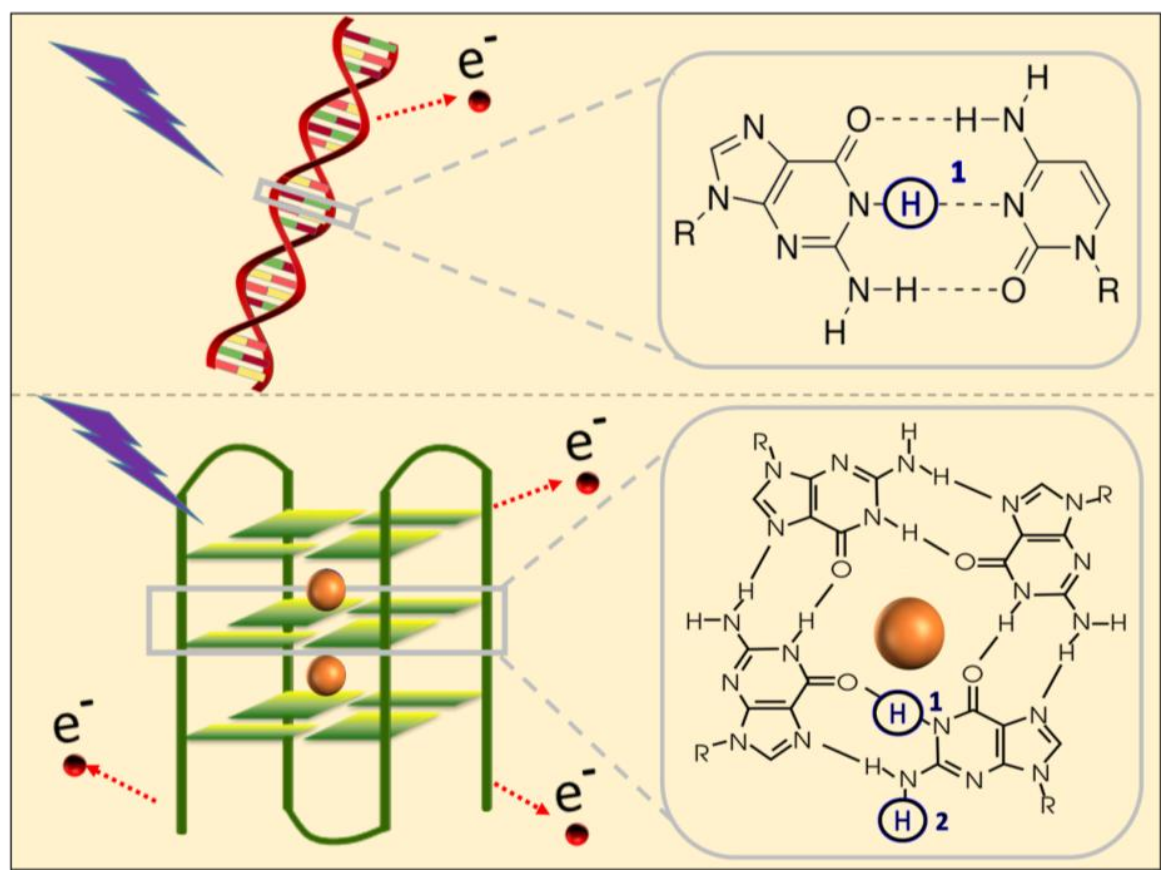




\section{Introduction}

UV radiation instigates oxidative damage to DNA in an indirect or a direct way. ${ }^{1}$ The former mechanism involves mediation of other molecules that absorb light and, subsequently, oxidize DNA through release of reactive oxygen species or photosensitized electron abstraction. In the latter, a photon directly absorbed by DNA provokes photo-ionization: an electron is ejected and a hole, that is a radical cation, is created. Regardless the mechanism and the initial location of the electron hole, after charge transfer, ${ }^{2-8}$ it reaches ultimately a guanine site. This happens because the guanine has the lowest oxidation potential compared to the other nucleobases, sugar and phosphate moieties composing DNA. ${ }^{9}$ The present Account describes guanine radical generation via low-energy photo-ionization and discusses their evolution.

As the vertical ionization potential (VIP) of the various DNA components is higher than 6.6 $\mathrm{eV}^{10,11}$ and excitation at the lowest absorption band (Figure 1a) is not expected to provoke onephoton ionization. Therefore, this phenomenon was studied by exciting at $193 \mathrm{~nm}(6.4 \mathrm{eV}) .{ }^{12-14}$ Yet, more than a decade ago, two studies reported electron ejection from DNA oligomers, both in aqueous solution ${ }^{15}$ and in the gas phase,${ }^{16}$ at much lower energy (266 nm, $\left.4.7 \mathrm{eV}\right)$; but these results appeared rather as a curiosity, connected with laser experiments. More recently, some of us showed that low-intensity continuous irradiation of purified genomic DNA at 554 and $295 \mathrm{~nm}$ gives rise not only to the well-known oxidation marker 8-oxo-7,8-dihydro-2'-deoxyguanosine (8-oxodG), but also to polyamine-guanine adducts. ${ }^{17}$ While 8 -oxodG can be produced by several pathways, including DNA-mediated singlet oxygen generation, ${ }^{18,19}$ the detected adducts are typical of a mechanism involving the guanine radical cation $\left(\mathbf{G}^{+}\right)^{\circ}$. In parallel, we have undertaken a study aiming at the quantitative characterization of the related primary species, ejected electrons and guanine radicals, by transient absorption spectroscopy using as excitation 
source $5 \mathrm{~ns}$ laser pulses at $266 \mathrm{~nm}$. Although nanosecond flash photolysis has been a laboratory tool for over half a century now, specific protocols had to be developed for these studies. The reason is that they require detection of very weak signals arising from DNA systems undergoing damage during the measurement.

Our photo-ionization experiments have also proven a unique tool to study the "intrinsic" reaction dynamics of radicals; the term "intrinsic" refers to the absence of any external additive other than the ingredients of the phosphate buffer used as a solvent, which do not react with $\mathbf{G}$ radicals. As guanine radical cations $\left(\mathbf{G}^{+}\right)^{\bullet}$ are known to be metastable undergoing either hydration or loss of a proton, ${ }^{20}$ the time-resolved spectra allow us to follow their transformation and evaluate the evolution of their populations. This is an important issue because deprotonated radicals give rise to lesions different from those stemming directly from $\left(\mathbf{G}^{+}\right)^{\cdot 1}{ }^{1}$ Consequently, the final type of oxidative damage depends, among others, on the population of $\left(\mathbf{G}^{+}\right)^{\bullet}$ that undergoes deprotonation.

Our studies revealed that the secondary DNA structure plays a key role both in the generation of guanine radicals by low-energy photo-ionization and in their evolution. Here, we discuss our previous studies on model duplexes ${ }^{21-24}$ and present new results on calf thymus (CT-DNA), showing that genomic DNA exhibits the same behavior as small size systems. In addition, we exemplify the behavior of G-quadruplexes (G4), whose building blocks are guanine tetrads (Conspectus Figure), by presenting new data obtained for Oxytricha G4. The latter, obtained by association of two single strands GGGGTTTTGGGG (OXY), ${ }^{25}$ confirm previous studies reported for monomolecular ${ }^{24,26,27}$ and tetramolecular systems. ${ }^{28-30}$ Our G4 are formed and studied in the presence of $\mathrm{Na}^{+}$or $\mathrm{K}^{+}$cations. These cations, are not only part 
of the bulk solvent; they are also located in the central cavity of $\mathbf{G 4}$, being constitutive elements of their structure. ${ }^{31}$ Therefore, when referring to G4, we always indicate the type of metal cations, for example: $\mathbf{O X Y} / \mathbf{N a}^{+}$and $\mathbf{O X Y} / \mathbf{K}^{+}$.

\section{Key experimental points}

Experimental details are given in the Supporting Information (SI-Section 1). Here, we simply highlight a few points that are crucial for these studies.

Materials. Both synthetic oligomers and genomic DNA were extensively purified. Moreover, the purity of the chemicals used for the preparation of the buffer was $99.99 \%$. This is an important requirement for avoiding photo-ionization of impurities, on the one hand, and their reaction with radicals, on the other. The buffer ( $\mathrm{pH} 7)$ was composed of an equimolar mixture of $\mathrm{MH}_{2} \mathrm{PO}_{4}$, and $\mathrm{M}_{2} \mathrm{HPO}_{4}$, where $\mathrm{M}^{+}$is either $\mathrm{Na}^{+}$or $\mathrm{K}^{+}$. In most experiments, their concentration was high $\left(0.15 \mathrm{~mol} \cdot \mathrm{L}^{-1}\right)$ so that to scavenge the ejected electrons, ${ }^{32}$ preventing their interaction with DNA. Furthermore, the corresponding high ionic strength helped stabilizing short duplexes. During the measurement, the solution was frequently renewed so that to avoid that successive laser pulses excite already damaged DNA; typically, for a complete study of a given system, 300-500 mL were needed.

Spectroscopic measurements. The challenge was to work with low-excitation intensities, leading to very weak transient absorption signals, so that to avoid two-photon ionization of the buffer, saturation effects in electron ejection (SI-Section 2), interaction among radicals and minimize two-photon ionization of DNA. Transient absorption signals were optimized by using orthogonal exciting and probing beams whose path-lengths though the sample were, respectively, $0.1 \mathrm{~cm}$ and $1 \mathrm{~cm}$; the absorbance of the solution on the excitation side was $0.25 \pm$ 0.02 , corresponding to a base concentration of the order of $10^{-4} \mathrm{~mol} \cdot \mathrm{L}^{-1}$. The incident pulse 
intensity did not exceed, depending on the system, $2 \times 10^{6}$ or $3 \times 10^{6} \mathrm{~W} \cdot \mathrm{cm}^{-2}$ (SI-Section 2 ). In this way, the transient absorption decays are not influenced by the incident excitation intensity (Figure S2). Finally, the concentration of photons absorbed in the probed volume of the studied solution $[\mathrm{h} v]$ was determined using a NIST traceable power-meter and counterchecked via an actinometer (SI-Section 1). In all experiments, the concentration of generated radicals was three orders of magnitude lower than the base concentration.

\section{Characterization of ejected electrons}

Electrons issued from photo-ionization in aqueous solution are known to be hydrated $\left(\mathrm{e}_{\mathrm{hyd}}{ }^{-}\right)$ and exhibit a broad absorption band peaking around $720 \mathrm{~nm}$. Such a band is indeed present in the transient absorption spectrum recorded for CT-DNA at 30 ns, corresponding to the 30 -ns time resolution of our experimental setup (Figure 1b). Based on this property and taking into account a maximum molar absorption coefficient $\varepsilon$ of $19700 \mathrm{~mol}^{-1} \cdot \mathrm{L} \cdot \mathrm{cm}^{-1}$ reported for $\mathrm{e}_{\text {hyd }}$, we quantify the ejected electrons by probing their transient absorption on the sub-microsecond timescale.
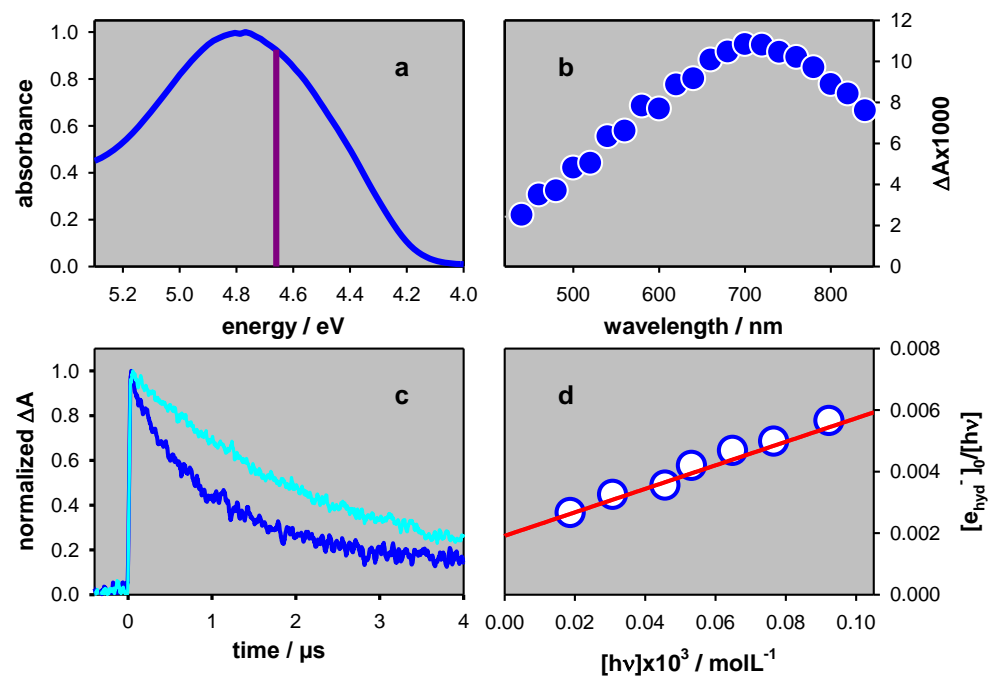
Figure 1. CT-DNA (blue) in phosphate buffer $\left(0.015 \mathrm{~mol} \cdot \mathrm{L}^{-1} \mathrm{NaH}_{2} \mathrm{PO}_{4} / 0.015 \mathrm{~mol} \cdot \mathrm{L}^{-1} \mathrm{Na}_{2} \mathrm{HPO}_{4}\right)$. (a) Steady-state absorption spectrum; the vertical violet line indicates the excitation wavelength at $266 \mathrm{~nm}$.

(b) Transient absorption spectrum recorded at $30 \mathrm{~ns}$ with incident excitation intensity of $2.0 \times 10^{6} \mathrm{~W} \cdot \mathrm{cm}^{-2}$.

(c) Normalized transient absorption decay at $700 \mathrm{~nm}$; it is compared with the corresponding signal obtained under the same conditions for a short duplex (cyan; S1•S2 ${ }^{24}$ Table 1). (d) Ionization curve; [hv] and $\left[\mathrm{e}_{\mathrm{hyd}}\right]_{0}$ denote, respectively, the concentration of absorbed photons per laser pulse and the initial concentration of hydrated electrons; the red line is the fit with a linear model function. (b-d): argon saturated solutions.

In our experimental conditions, $\mathrm{e}_{\text {hyd }}{ }^{-}$disappear within a few $\mu$ s, reacting with $\mathrm{H}_{2} \mathrm{PO}_{4}{ }^{-}$ions ${ }^{32}$ present in high concentration. Their decay rate is the same for all the small DNA systems (Figure S3), depending only on the buffer concentration. In contrast, for the same buffer concentration, $\mathrm{e}_{\text {hyd }}{ }^{-}$stemming from CT-DNA decay significantly faster (Figures 1c), revealing the existence of additional reaction paths, involving DNA. ${ }^{34}$ Such reactions could be favored by the flexibility of the very long duplex, ${ }^{35}$ increasing locally the encounter probability between nucleic acid components and $\mathrm{e}_{\text {hyd }}{ }^{-}$produced by the same helix.

Although we use low excitation intensities, two-photon ionization, resulting from absorption of a second photon by a long-lived electronic excited state, cannot be completely prevented. To disentangle one- and two-photon events, we vary the excitation intensity, thus changing the concentration of absorbed photons. Subsequently, we plot $\left[\mathrm{e}_{\mathrm{hyd}}{ }^{-}\right]_{0} /[\mathrm{hv}]$ versus $[\mathrm{hv}]$, obtaining the so-called ionization curve (Figure 1d). The determination of $\left[\mathrm{e}_{\mathrm{hyd}}{ }^{-}\right]_{0}$ is illustrated in Figure S3: the $e_{\text {hyd }}{ }^{-}$decay is fitted with a model function $A_{1}+A_{0} \exp \left(-t / \tau_{1}\right)$; subsequently, $\left[e_{h y d}{ }^{-}\right]_{0}$ is obtained from the $\mathrm{A}_{0}$ value using the corresponding molar absorption coefficient. The fit of the experimental points in Figure 1d with a linear function $\left[\mathrm{e}_{\mathrm{hyd}}{ }^{-}\right]_{0} /[\mathrm{h} v]=\varphi_{1}+\alpha[\mathrm{h} v]$ provides the quantum yield for one-photon ionization $\varphi_{1}$, while the contribution of two-photon ionization $\alpha[h v]$ is given by the slope of the curve.

We did not detect one-photon ionization $\left(\varphi_{1} \leq 3 \times 10^{-4}\right)$ for mononucleotides and small single strands characterized by poor base stacking, such as the 6-mer telomere repeat TTAGGG ${ }^{26}$ or 
thymine single strands $(\mathrm{dT})_{20} \cdot{ }^{15}$ In contrast, for adenine single strands, known to adopt a helical structure, a $\varphi_{1}$ value of $(1.0 \pm 0.1) \times 10^{-3}$ was found. ${ }^{21}$ Those determined for various duplexes and G4 are gathered on Table 1 . In the case of duplexes, $\varphi_{1}$ ranges from $1.0 \times 10^{-3}$ to $2.0 \times 10^{-3}$. $^{21-24}$ This concerns also icosamers containing only adenines and thymines in alternating ${ }^{22}$ or homopolymeric sequence. ${ }^{21}$ Clearly higher values, which may approach $10^{-2}$, are observed for G4. ${ }^{24,26,28-30}$ In this family, topology and molecularity seem to play a rather minor role: the behavior of tetramolecular $\left(\mathbf{T G}_{\mathbf{4}} \mathbf{T}\right)_{4} / \mathbf{N a}^{+}$and monomolecular $\mathbf{T E L 2 1} / \mathbf{N a}^{+}$systems is similar although their guanines adopt, respectively, parallel and antiparallel orientations in respect to the glyosidic bonds. ${ }^{26,28}$ In contrast, the metal cation used in the buffer in which $\mathbf{G 4}$ are formed and studied, has an important effect: the values found in the case of $\mathrm{K}^{+}$are significantly higher compared to $\mathrm{Na}^{+}$.

Table 1. Quantum yields $\left(\varphi_{1} \times 10^{3}\right)$ of one-photon ionization at $266 \mathrm{~nm}$ and guanine/adenine radical halflives (ms, in brackets) determined for duplexes and G4 in phosphate buffer.

\begin{tabular}{|c|c|c|c|c|c|c|}
\hline & $\mathrm{M}^{+}$ & CT-DNA & $(\mathrm{GC}) 5^{\circ}(\mathrm{GC}) 5^{\mathrm{a}}$ & $\mathbf{S} 1 \cdot \mathbf{S} 2^{\mathrm{b}}$ & $(\mathrm{AT})_{10^{\circ}}(\mathrm{ATT})_{10^{c}}^{\mathrm{c}}$ & $(\mathrm{A})_{20} \cdot(\mathrm{T})_{20}{ }^{\mathrm{d}}$ \\
\hline \multirow[t]{3}{*}{ duplexes } & $\mathrm{Na}^{+}$ & $\begin{array}{l}2.0 \pm 0.2 \\
(3.7 \pm 0.2)\end{array}$ & $\begin{array}{l}1.2 \pm 0.2^{23} \\
(2.5 \pm 0.2)\end{array}$ & $\begin{array}{c}2.1 \pm 0.4^{24} \\
(4 \pm 0.2)\end{array}$ & $\begin{array}{l}1.5 \pm 0.3^{22} \\
(2.0 \pm 0.1)\end{array}$ & $\begin{array}{l}1.1 \pm 0.3^{21} \\
(4.0 \pm 1.0)\end{array}$ \\
\hline & $\mathrm{K}^{+}$ & $\begin{array}{l}1.9 \pm 0.1 \\
(3.6 \pm 0.2)\end{array}$ & - & - & - & \\
\hline & $\mathrm{M}^{+}$ & TEL 21 $^{\mathrm{e}}$ & TEL25 $^{f}$ & $\mathbf{O X Y}^{\mathrm{g}}$ & $\left(\mathrm{TG}_{4} \mathrm{~T}\right)_{4}{ }^{\mathrm{h}}$ & \\
\hline \multirow[t]{2}{*}{ G4 } & $\mathrm{Na}^{+}$ & $\begin{array}{l}4.5 \pm 0.6^{26} \\
(6.7 \pm 0.5)\end{array}$ & $\begin{array}{l}5.2 \pm 0.3^{24} \\
(3.5 \pm 0.2)\end{array}$ & $\begin{array}{c}6.0 \pm 0.2 \\
(4.1 \pm 0.2)\end{array}$ & $\begin{array}{l}3.5 \pm 0.5^{28} \\
(6.0 \pm 0.5)\end{array}$ & \\
\hline & $\mathrm{K}^{+}$ & $\begin{array}{l}9.4 \pm 0.1^{27} \\
(1.4 \pm 0.1)\end{array}$ & - & $\begin{array}{c}7.9 \pm 0.1 \\
(2.1 \pm 0.2)\end{array}$ & $\begin{array}{c}8.1 \pm 0.5^{29,30} \\
(3.1 \pm 0.2)\end{array}$ & \\
\hline
\end{tabular}

\footnotetext{
a) alternating guanine-cytosine; ${ }^{\text {b) }} \mathrm{S} 1$ : 5'-CGTACTCTTTGGTGGGTCGGTTCTTTCTAT-3'/

S2: 3'-GCATGAGAAACCACCCAGCCAAGAAAGATA-5', c) alternating adenine-thymine; ${ }^{\text {d) }}$ homopolymeric adeninethymine; ${ }^{\text {e) }}$ monomolecular: GGG(TTAGGG) $)_{3}$; ${ }^{\text {f) }}$ monomolecular: TAGGG(TTAGGG) $)_{3}$ TT; ${ }^{\text {g) }}$ bimolecular: $2 \times($ GGGGTTTTGGGG $){ }^{\text {h) }}$ tetramolecular: $4 \times($ TGGGGT $)$.
} 


\section{Evidence for a non-vertical photo-ionization mechanism?}

The effect of metal ions on the propensity of $\mathbf{G} 4$ to undergo low-energy photo-ionization points towards non-vertical electron ejection. This conclusion was drawn, in particular, by comparing $\left(\mathbf{T G}_{\mathbf{4}} \mathbf{T}\right)_{4} / \mathrm{Na}^{+}$and $\left(\mathbf{T G}_{\mathbf{4}} \mathbf{T}\right)_{4} / \mathbf{K}^{+},{ }^{28-30}$ in which the static arrangement of nucleobases is the same. Although the VIP of these two systems, computed by quantum chemistry methods, are practically identical, ${ }^{19}$ we observe a twofold increase in $\varphi_{1}$ upon replacing $\mathrm{Na}^{+}$by $\mathrm{K}^{+}$cations. Hence, a complex mechanism, associated with the excited state relaxation, was proposed. The first step implies population of an excited charge transfer (CT) state, either between a thymine and a guanine or between two guanines. In a second step (charge separation), the positive charge is trapped by the guanine core, where it may be delocalized, ${ }^{11,36-38}$ while the negative one is born by a thymine. This process is tentatively described as:

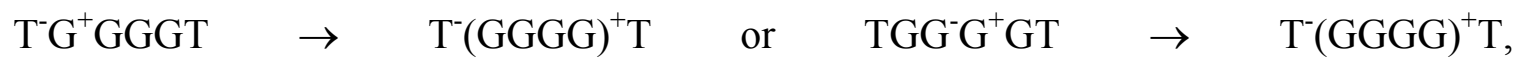
where the radical sign was omitted for simplicity. Finally, electron ejection, assisted by conformational motions, occurs from the thymine anion, whose VIP in the gas phase is lower than $0.1 \mathrm{eV} \cdot{ }^{39}$ In the frame of such a scenario, $\mathrm{Na}^{+}$cations, smaller and more mobile than $\mathrm{K}^{+},{ }^{40}$ allow the geometrical arrangement accompanying stabilization of CT states, ${ }^{41}$ and, hence, reducing the probability of charge separation.

Population of excited CT states both in duplexes ${ }^{42-44}$ and $\mathbf{G 4}^{41,45,46}$ is well documented. The occurrence of charge separation along DNA strands has also been reported. ${ }^{47}$ The higher $\varphi_{1}$ in G4, could be due to more efficient trapping of the positive charge within stacked guanine tetrads (Conspectus Figure); in this way, charge separation becomes more efficient, increasing the probability of electron ejection from a base located outside the guanine core. Another important difference is that guanine tetrads interact directly with dehydrated cations. ${ }^{31}$ Such interaction is 
not encountered in duplexes, explaining why the $\varphi_{1}$ of CT-DNA is not significantly different in presence of $\mathrm{Na}^{+}$or $\mathrm{K}^{+}$cations in contrast to $\mathbf{G} 4$ scaffolds (Table 1).

In contrast to the vertical ionization process, whose quantum yield decreases rapidly when moving away from the VIP, the proposed mechanism is expected to be operative over a broader range of excitation energy, provided that $\mathrm{CT}$ states are populated and charge separation achieved. This is supported by the fact that similar quantum yields for 8-oxodG formation were obtained for UVC and UVB irradiation. ${ }^{17}$ Furthermore, strand breaks were observed upon UVA irradiation, $^{48}$ where the weakly absorbing excited states ${ }^{49,50}$ have partial charge transfer character. $^{50,51}$

\section{Radicals}

General considerations. The spectral fingerprints of radicals are determined after the decay of $\mathrm{e}_{\text {hyd }}{ }^{-}$. We identify them by comparison with the corresponding spectra reported for monomeric guanosine derivatives (Figure 2a). We checked experimentally the validity of this comparison for $\left(\mathbf{G}^{+}\right)^{\bullet}$, for which stronger interactions with neighboring bases are expected than for deprotonated radicals, because of the presence of a positive charge. To this end, we recorded time-resolved spectra of $\mathbf{G} 4$ at pH3, where deprotonation of $\left(\mathbf{G}^{+}\right)^{\bullet}$ is hindered. ${ }^{12}$ This is shown in Figure $2 b$ in the case of $\left(\mathbf{T G}_{\mathbf{4}} \mathbf{T}\right)_{4} / \mathbf{N a}^{+}:{ }^{28}$ the agreement with the monomeric radical spectrum is satisfactory in the 450-700 nm domain, not only regarding the spectral shape but also the extinction coefficients determined by dividing the observed transient absorption $\Delta \mathrm{A}$ at $2.5 \mu \mathrm{s}$ by $\left[\mathrm{e}_{\text {hyd }}{ }^{-}\right]_{0}$, which corresponds to that of generated electron holes. As predicted by quantum chemistry calculations, lower $\varepsilon$ values were found for the band at $400 \mathrm{~nm} \cdot{ }^{26,28}$ Below this wavelength, it was impossible to determine reliable spectra due to strong photodegradation occurring in acidic solutions during the measurements. 

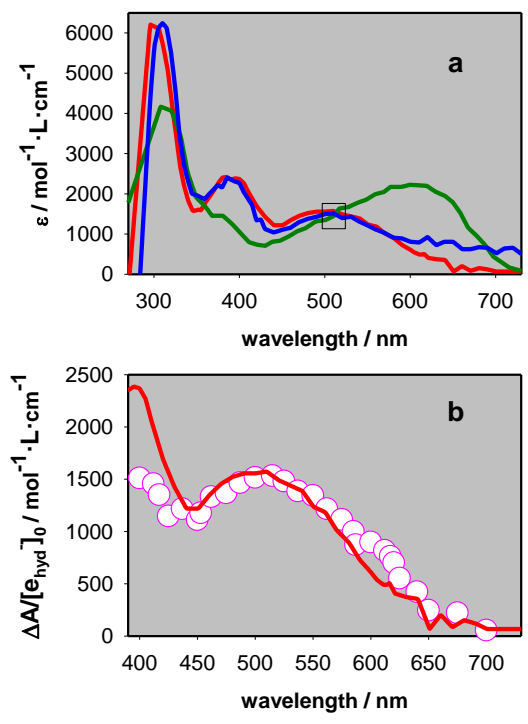

Figure 2. Reference monomeric $\mathbf{G}$ radicals. Absorption spectra of monomeric radical cation $\left(\mathbf{G}^{+}\right)^{\bullet}($ red $){ }^{12}$ and deprotonated $(\mathbf{G}-\mathrm{H} 1)^{\bullet}$ radical (blue) ${ }^{12}$ and $(\mathbf{G}-\mathrm{H} 2)^{\bullet}$ radicals (green), ${ }^{52}$ resulting, respectively, from loss of the protons at position 1 or 2 (Conspectus Figure); the black square indicates the spectral region where the $\varepsilon$ values of all three radicals are similar $\left(\Delta \varepsilon<100 \mathrm{~mol}^{-1} \cdot \mathrm{L} \cdot \mathrm{cm}^{-1}\right){ }^{27}$ (b) Transient absorption spectrum obtained for $\left(\mathbf{T G}_{\mathbf{4}} \mathbf{T}\right)_{4} / \mathbf{N a}^{+}$in $\mathrm{pH} 3$ at $2.5 \mu$ s (circles); $\Delta \mathrm{A}$ is divided by $\left[\mathrm{e}_{\text {hyd }}{ }^{-}\right]_{0}$, determined in the same experiment; the spectrum of the monomeric $\left(\mathbf{G}^{+}\right)^{\bullet}(\text { red })^{28}$ is represented with $\varepsilon$ values.

In Figure 2a, we remark the existence of a quasi-isosbestic point around 500-515 nm. Thus, regardless the radical evolution in a given system, the total radical concentration at time $t,[R]_{t}$, may be obtained from the $\Delta \mathrm{A}$ value at this wavelength range. We found that for all the examined systems, $[\mathrm{R}]_{\mathrm{t}}$ at $3 \mu \mathrm{s}$ matches the concentration of hydrated ejected electrons, corresponding to the initially created electron holes $\left([\mathrm{R}]_{0}=\left[\mathrm{e}_{\text {hyd }}{ }^{-}\right]_{0}\right)$. This means that only a small fraction of the radical population, associated with our experimental error (5-10\%, depending on the system), reacts at earlier times. Consequently, the survival probability of the total radical population is given by the decays recorded at the quasi-isosbestic point, setting $\Delta \mathrm{A}$ at $3 \mu$ s equal to 1 . The survival probabilities determined for CT-DNA and OXY/K ${ }^{+}$are shown in Figure 3. 

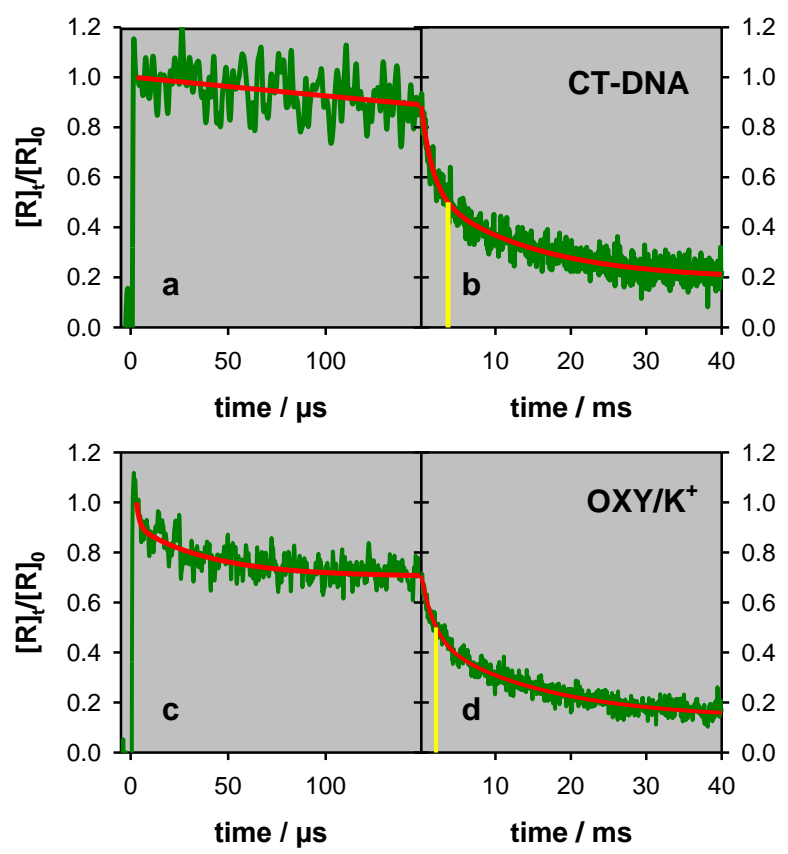

Figure 3. Survival probability of the total $\mathbf{G}$ radical population in CT-DNA (a and b) and OXY/K $\mathbf{K}^{+}$(c and d). Experimental decays (green) recorded at $500 \mathrm{~nm}$ (a and b) and $512 \mathrm{~nm}$ (c and d) are fitted with multiexponential functions (red). The vertical yellow lines indicate $\tau_{1 / 2}$.

The radical half-lives $\tau_{1 / 2}$, that is the time at which $[\mathrm{R}]_{\mathrm{t}} /[\mathrm{R}]_{0}=0.5$, are of a few milliseconds in both duplexes and G4 (Table 1). The $\tau_{1 / 2}$ found for the duplex S1•S2 (4 ms) is twice as long than those determined for the two parent single strands. ${ }^{24}$ A similar increase in $\tau_{1 / 2}$ upon base-pairing is observed for adenine radicals in A-tracts. ${ }^{21}$ Larger conformational motions in single strands probably allow the systems to adopt more rapidly reactive conformations, increasing the reaction rate. Finally, we remark in Table 1 that $\tau_{1 / 2}$ of $\mathbf{G 4}$ is much shorter in the presence of $\mathrm{K}^{+}$ compared to $\mathrm{Na}^{+}$. The reasons underlying this effect are not clear yet.

CT-DNA. The transient absorption spectrum determined for CT-DNA at $50 \mu \mathrm{s}$ is shown in Figure 4 a. It strongly resembles that of $(\mathbf{G}-\mathbf{H 1})^{\bullet}$ radical reported for dGMP; ${ }^{12}$ the agreement is quantitative, reproducing the $\varepsilon$ values. However, as in the case of short duplexes, ${ }^{24}$ the relative intensity at $700 \mathrm{~nm}$ in respect to that at $500 \mathrm{~nm}(\sim 0.6)$ is higher than that characterizing the 
monomer spectrum ( 0.4). Such an enhanced absorption at the red part is predicted by quantum chemistry calculations for guanine radicals within G-C pairs. ${ }^{23,53}$ It was shown, in particular, that when the proton is transferred to the bulk water instead of cytosine, a weak intensity band appears around $650 \mathrm{~nm}^{53}$

We determined the deprotonation dynamics in CT-DNA by monitoring the transient absorption at $700 \mathrm{~nm}$ because only (G-H1) ${ }^{\bullet}$ absorbs at this wavelength (Figure 2a). To this end, we eliminated the much stronger absorption of $\mathrm{e}_{\text {hyd }}$ - using efficient electron scavengers $\left(\mathrm{N}_{2} \mathrm{O}\right.$ and $\mathrm{NO}_{3}{ }^{-}$). ${ }^{32}$ Under these conditions, we observed a rise which is completed within $\sim 2 \mu$ s (Figure $4 \mathrm{~b})$; it can be approximated with a monoexponential function with a time constant of $0.3 \mu \mathrm{s}$, in line with the fast deprotonation rate obtained for short duplexes using pulse radiolysis. ${ }^{54}$ Subsequently, we found that the signal starts decaying slowly on the $\mu$ s time-scale (Figure 4c), with a rate similar to that at $500 \mathrm{~nm}$ (Figure 3a). The indirect oxidation study reported instead a slow rise corresponding to a time constant of $7 \mu \mathrm{s} .{ }^{54}$ This discrepancy probably arises from anisotropic reaction kinetics between the oxidant and DNA bases in the duplex, as discussed in detail in reference 24 . 

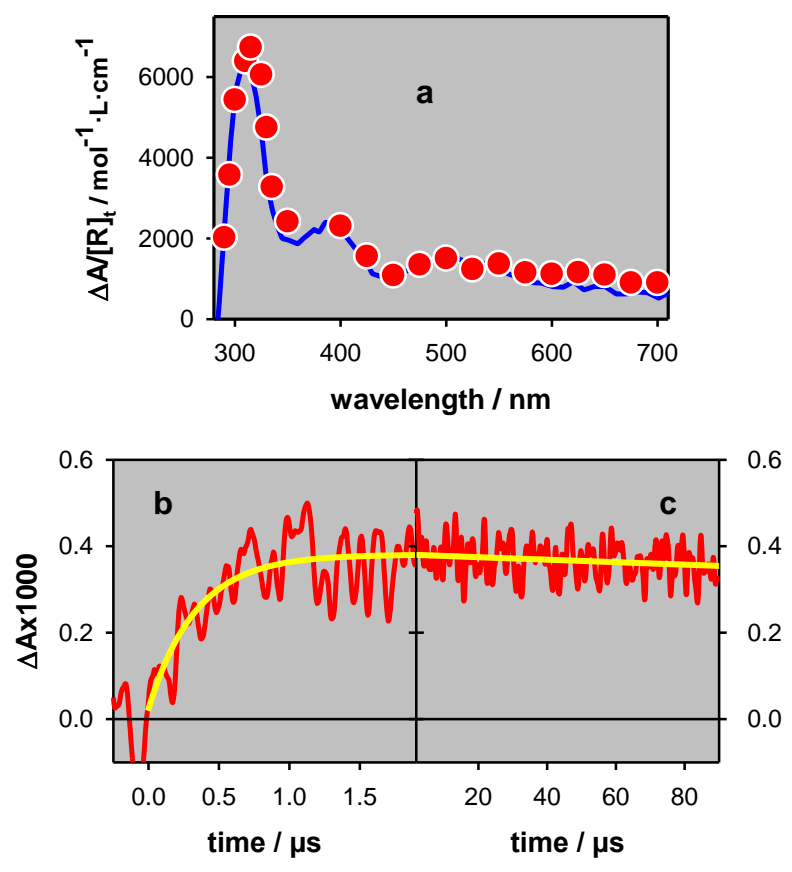

Figure 4. Guanine radicals in CT-DNA. (a) Transient absorption spectrum determined for aerated solutions at $50 \mu \mathrm{s}$ (red circles); $\Delta \mathrm{A}$ was divided by $[\mathrm{R}]_{\mathrm{t}}$. The blue line corresponds to the deprotonated radical of dGMP attributed to $(\mathbf{G}-\mathrm{H} 1)^{\circ}$, represented with $\varepsilon$ values. ${ }^{12}$ (b and c) Transient absorption signals recorded for $\mathrm{N}_{2} \mathrm{O}$ saturated solutions at $700 \mathrm{~nm}$; yellow lines are derived from fits with monoexponential rise/decay; incident excitation intensity: $2.0 \times 10^{6} \mathrm{~W} \cdot \mathrm{cm}^{-2}$.

The $\varepsilon$ values of the $(\mathbf{G}-\mathrm{H} 1)^{\bullet}$ at $700 \mathrm{~nm}$ have not been determined experimentally for duplexes. However, the spectral shape in Figure $4 \mathrm{a}$, in analogy with the computed spectra, ${ }^{53}$ as well as the extent of rise in Figure $4 b$, strongly suggest that the quasi-entire population of $\left(\mathbf{G}^{+}\right)^{\bullet}$ undergoes deprotonation within $2 \mu \mathrm{s}$.

Radicals in G4. Going from duplexes to four-stranded structures, the spectra recorded at $3 \mu \mathrm{s}$ exhibit a broad absorption band in the visible, attributed to the coexistence of $\left(\mathbf{G}^{+}\right)^{\bullet}$ and $(\mathbf{G}-\mathrm{H} 2)^{\bullet}$ radicals. As the hydrogen at position 1 of guanines (Conspectus Figure) is engaged in hydrogen bonding within the tetrads, deprotonation occurs at position 2 . The existence of long-lived $\left(\mathbf{G}^{+}\right)^{\bullet}$ in $\mathbf{G} 4$ and their deprotonation toward $(\mathbf{G}-\mathrm{H} 2)^{\bullet}$, was reported in a study performed via photosensitization. ${ }^{55}$ In our studies, we quantified the population of these long-lived $\left(\mathbf{G}^{+}\right)^{\bullet}$. To 
this end, we reconstructed the time-resolved spectra with linear combinations of the spectra of monomeric radicals. An example is shown in Figure 5a, where the reconstruction of the transient absorption spectrum obtained for $\mathbf{O X Y} / \mathbf{K}^{+}$at $3 \mu$ s is presented. The fraction of the radical population persisting as electron holes at $3 \mu$ s ranges from 0.25 to 0.50 , depending on the $\mathbf{G} 4$ structure. $^{24,26-30}$

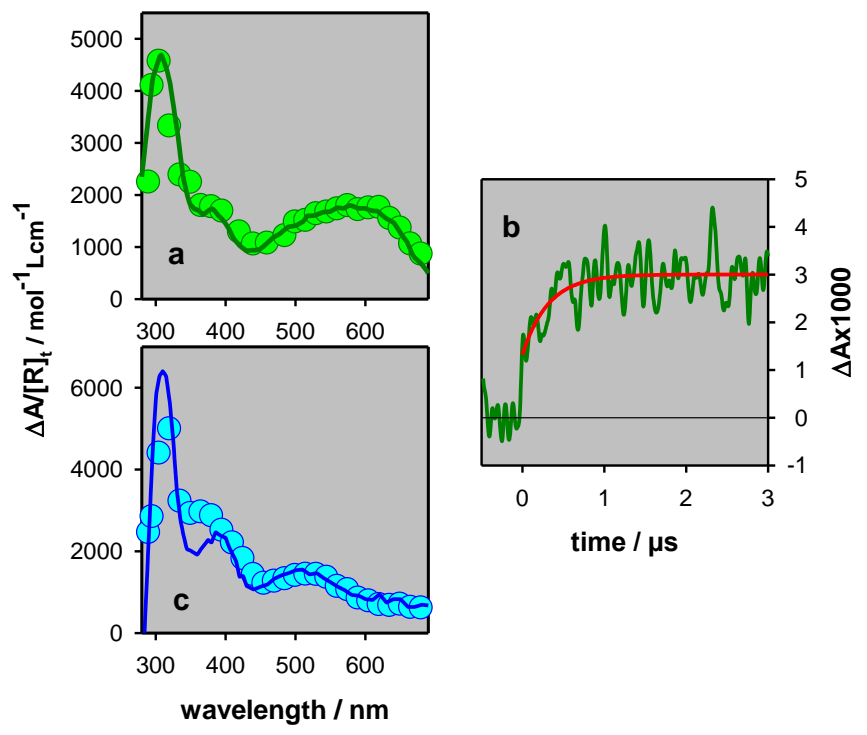

Figure 5. G radicals in $\mathbf{O X Y} / \mathbf{K}^{+}$. Transient absorption spectra (circles) at $3 \mu \mathrm{s}\left(\mathrm{a},[\mathrm{R}]_{\mathrm{t}}=[\mathrm{R}]_{0}\right)$ and $10 \mathrm{~ms}$ $\left(c,[R]_{t}=0.3[R]_{0}\right)$ determined for aerated solutions; the green line in (a) is a linear combination of the spectra corresponding to $\left(\mathbf{G}^{+}\right)^{\bullet}(30 \%)^{12}$ and the $(\mathbf{G}-\mathrm{H} 2)^{\bullet}(70 \%)^{52}$ radicals of monomeric guanosine; the blue line in (c) corresponds to the spectrum $(\mathbf{G}-\mathrm{H} 1)^{\circ}$ of dGMP. ${ }^{12}$ (b) Transient absorption trace at $620 \mathrm{~nm}$ recorded for $\mathrm{N}_{2} \mathrm{O}$ saturated solutions (green); the red line corresponds to the fit with a monoexponential rise. Incident excitation intensity: $2.0 \times 10^{6} \mathrm{~W} \cdot \mathrm{cm}^{-2}$.

The $\left(\mathbf{G}^{+}\right)^{\bullet} \rightarrow(\mathbf{G}-\mathrm{H} 2)^{\bullet}$ deprotonation in $\mathbf{G} 4$ spans several decades of time. It can be observed by the rise of the transient absorption signal at $600-620 \mathrm{~nm}$, where the difference between the two radical spectra is particularly important (Figure 2a). The fastest part (Figure 5b) is probably related with outer tetrads that are most exposed to bulk water. Completion of the $\left(\mathbf{G}^{+}\right)^{\bullet}$ deprotonation ranges from few decades of microseconds in tetramolecular $\mathbf{G} 4^{28-30}$ to a few milliseconds in TEL21/Na ${ }^{+}{ }^{26}$ In most cases, $\left(\mathbf{G}^{+}\right)^{\bullet} \rightarrow(\mathbf{G}-\mathrm{H} 2)^{\bullet}$ deprotonation is followed by $(\mathbf{G}-$ 
$\mathrm{H} 2)^{\bullet} \rightarrow(\mathbf{G}-\mathrm{H} 1)^{\bullet}$ tautomerization. ${ }^{24,26-28}$ An example is shown in Figure 5c, where the $\mathbf{O X Y} / \mathbf{K}^{+}$ spectrum at $10 \mathrm{~ms}$ in the visible spectral domain corresponds to that of $(\mathbf{G}-\mathrm{H} 1)^{\bullet}$ in a concentration equal to $30 \%$ of $[R]_{0}$ (Figure $3 \mathrm{~d}$ ). Below $400 \mathrm{~nm}$ we observe the fingerprint of an unknown reaction intermediate, also detected for monomolecular $\mathbf{G 4}{ }^{24,27}$ Tautomerization is not observed in (TG4T) $)_{4} / \mathbf{K}^{+}$, where $(\mathbf{G}-\mathrm{H} 2)^{\bullet}$ radicals react rapidly through some other, currently unexplored, reaction path. ${ }^{20}$

\section{Correlation with final lesions}

Although the present study deals with primary processes, our findings shed some light upon final lesions. For example, the formation of 8-oxodG, known to stem from hydration of $\left(\mathbf{G}^{+}\right)^{\bullet}$, is in competition with the deprotonation process. Consequently, the level of 8 -oxodG will be determined by the relative rates of these two reactions. This effect is illustrated by a comparative study on TEL21/Na ${ }^{+}$and $\left(\mathbf{T G}_{4} \mathbf{T}\right)_{4} / \mathbf{N a}^{+}$, for which 8-oxodG was analyzed by high performance liquid chromatography coupled to mass spectrometry following irradiation at $266 \mathrm{~nm} .^{26,28}$ The quantum yield for 8-oxodG formation $\varphi_{\mathrm{ox}}$ was found to be $3.2 \times 10^{-4}$ for TEL21/Na ${ }^{+}$and lower than $10^{-5}$ for (TG4T) $)_{4} / \mathbf{N a}^{+}$. For the former system, in which part of the $\left(\mathbf{G}^{+}\right)^{\bullet}$ population survives on the millisecond time-scale, $\varphi_{\mathrm{ox}}$ represents $7 \%$ of the photo-ionization quantum yield $\phi_{1}$. Deprotonation is much faster in (TG4T) $)_{4} / \mathbf{N a}^{+}$, being completed within $20 \mu$ s, thus rendering the reaction path leading to 8-oxodG less probable $\left(\varphi_{\mathrm{ox}} / \varphi_{1}<0.003\right)$.

As we mentioned above, we found that the largest part of the initially generated $\left(\mathbf{G}^{+}\right)^{\bullet}$ population in the examined systems undergoes deprotonation. Therefore, we expect the major oxidative damage to arise from deprotonated radicals. As neither the radical concentration nor the decay patterns are affected by the buffer concentration (Figure S4a), we conclude that their 
reactions do not involve buffer ingredients. Furthermore, no significant difference was found between the transient signals recorded for aerated and argon saturated solutions (Figure S4b). The latter observation rules out formation of imidazolone and oxazolone, known to be formed from $(\mathbf{G}-\mathrm{H} 1)^{\bullet}$ radicals exclusively in the presence of oxygen. In contrast, the formation of oxidized bases and strand breaks are not affected by oxygen. ${ }^{56,57}$ In the case of plasmid DNA, the yield of oxidized bases was found to be 20 -fold higher than strand breaks. ${ }^{56}$ Interestingly, strand breaks were detected in plasmid DNA following direct absorption of low-energy radiation, ${ }^{48}$ but quantum yields were not determined.

Our studies, performed without external additives, show that deprotonated radicals react mostly on the millisecond time-scale in an "intrinsic" way. This means that $\mathbf{G}$ radicals disappear reacting with other parts of the nucleic acids and/or water molecules. Evidently, the reaction path may be altered by the presence of other chemical species in their vicinity, as happens in cells. The outcome will depend on the relative reaction rates of "extrinsic" and "intrinsic" paths, the final lesions being controlled by the faster process. The same reasoning is valid for the photoejected electrons which, in our experimental conditions appear to interact with genomic DNA, but may be scavenged by other chemical species present in biological conditions.

\section{Conclusions and Outlook}

The studies presented in this Account have challenged two widely accepted ideas regarding DNA damage and lead to important questions.

Firstly, we showed that direct absorption of low-energy UV radiation by DNA does generate guanine radicals. In the case of genomic DNA, the quantum yield of this process at $266 \mathrm{~nm}$ $\left(2.0 \times 10^{-3}\right)$ is similar to the sum of the quantum yields of all types of dimeric pyrimidine 
photoproducts, both cyclobutane dimers and (6-4) adducts, following $254 \mathrm{~nm}$ irradiation $\left(1.3 \times 10^{-3}\right) .^{58}$ The generation of guanine radicals via direct photo-oxidation, occurring without the mediation of photosensitizers, has important repercussion on preventive measures: administration of antioxidants to protect DNA damage from low-energy UVB and UVA radiation, although proved efficient for indirect damage, cannot be operative for direct UVinduced radical generation.

Secondly, we demonstrated that 8-oxodG, a widely used oxidation marker, is not representative of the extent of oxidative damage when this is triggered by generation of radical cations. In this case, the major oxidative lesions should stem from deprotonated radicals and be context dependent, because of the long lifetime of these species. Photo-ionization quantum yields provide an estimation for the expected total amount of oxidative lesions. In association with analytical methods, they could contribute to quantify the various types of final reaction products and, possibly, lead to characterization of new ones. For example, the lesions resulting from (G$\mathrm{H} 2)^{\bullet}$ radicals, detected in all $\mathbf{G} 4$ structures, remain to be identified.

To explain photo-ionization by low-energy UV photons, we proposed a complex path, encompassing recent knowledge of the electronic excited states in DNA. This hypothesis is supported by a series of observations associating photo-ionization with DNA secondary structure. It also explains the detection of 8-oxodG and strand breaks after UVB and UVA irradiation of DNA. A non-vertical mechanism is also suggested by electron detachment studies on DNA polyanions in the gas phase ${ }^{59}$ However, more effort is needed for its validation, fully characterizing the various steps that lead to electron photo-detachment and assessing the electronic, geometrical and dynamical factors that enter in play. We hope that this Account will 
motivate theoretical studies which will explore this phenomenon in detail and provide a comprehensive picture.

An important question concerns the sites on which oxidative lesions are formed. This issue was tackled by a photo-sensitization study on telomeric $\mathbf{G 4}{ }^{60}$ It would be interesting to explore whether the reactive sites created by external oxidants are similar to those generated through direct UV-induced oxidation.

Finally, beyond the biological issues discussed above, the larger propensity of $\mathbf{G 4}$ to undergo low-energy photo-ionization, compared to duplex DNA, deserves attention. G4 structures appear appealing for applications in the field of nanotechnology. ${ }^{61}$ Their behavior as molecular wires, connected to external electric circuits, was reported. ${ }^{62,63}$ But so far, their properties as photoconductors have been not explored. In this respect, our results could have an impact on the design of optoelectronic devices inspired by four-stranded scaffolds, potentially containing other functional groups.

ASSOCIATED CONTENT

Supporting Information: Experimental Details; Saturation Effect; Additional Figures.

\section{AUTHOR INFORMATION}

\section{Corresponding Author}

E-mail: Dimitra.markovitsi@cea.fr

ORCID : 0000-0002-2726-305X

\section{Biographies}


Mr. Evangelos Balanikas graduated from the National Technical University of Athens (Greece) in 2017. He currently works as early stage researcher in the frame of the European Training Network "LighDyNAmics: DNA as a training platform for photodynamic processes in soft materials". He prepares a $\mathrm{PhD}$ thesis on the UV-induced processes in G-quadruplexes.

Dr. Akos Banyasz obtained his PhD degree in Physical-Chemistry at the Eötvös Loránd University (Hungary) in 2006. He joined the CNRS in 2008 to investigate DNA photophysics and photochemistry induced by UV irradiation. He has expertise in time-resolved spectroscopy, from femtoseconds to milliseconds. Beyond DNA, his current research interests involve nonlinear spectroscopy and nonlinear 3D-printing.

Dr. Thierry Douki was trained in organic synthesis and joined the French Atomic Energy Commission in 1993. His research work is devoted to DNA damage and repair. He combines mechanistic studies on model systems with in vitro studies through development of specific analytical tools. The studied genotoxic agents include pollutants, oxidative stress and UV radiation.

Dr. Gérard Baldacchino obtained his PhD degree (1994) and Habilitation (2004) at the University Paris-Sud in the field of Molecular Chemistry. He joined the French Atomic Energy Commission in 1994, focusing his research on chemical effects induced by various types of radiation in aqueous systems. He developed sensitive transient absorption and fluorescence techniques to investigate complex radical mechanisms.

Dr. Dimitra Markovitsi received her $\mathrm{PhD} /$ Habilitation in Chemistry from the "Louis Pasteur" University (Strasbourg) in 1983. She joined the CNRS in 1981, where she is currently research director. She has expertise in photophysics and photochemistry, with emphasis on 
interchromophore interactions. During the past two decades she focused her efforts on the study of UV-induced processes in DNA, from photon absorption to excited state relaxation and reaction dynamics.

\section{Funding Sources}

The authors thank the European Programme H2020 MSCA ITN [grant No. 765266 LightDyNAmics project] and the French National Research Agency (N ANR-12-BS08-000101, OPHID project).

\section{REFERENCES}

(1) Cadet, J.; Douki, T. Formation of UV-induced DNA damage contributing to skin cancer development. Photochem. \& Photobiol. Sci. 2018, 17, 1816-1841.

(2) Lewis, F. D.; Young, R. M.; Wasielewski, M. R. Tracking Photoinduced Charge Separation in DNA: from Start to Finish. Acc. Chem. Res. 2018, 51, 1746-1754.

(3) Angelov, D.; Spassky, A.; Berger, M.; Cadet, J. High-intensity UV laser photolysis of DNA and purine 2'-deoxyribonucleosides: Formation of 8-oxopurine damage and oligonucleotide strand cleavage as revealed by HPLC and gel electrophoresis studies. J. Am. Chem. Soc. 1997, 119, 11373-11380.

(4) Meggers, E.; Michel-Beyerle, M. E.; Giese, B. Sequence dependent long range hole transport in DNA. J. Am. Chem. Soc. 1998, 120, 12950-12955.

(5) Saito, I.; Nakamura, T.; Nakatani, K.; Yoshioka, Y.; Yamaguchi, K.; Sugiyama, H. Mapping of the hot spots for DNA damage by one-electron oxidation: Efficacy of GG doublets and GGG triplets as a trap in long-range hole migration. J. Am. Chem. Soc. 1998, 120, $12686-12687$. 
(6) Kanvah, S.; Joseph, J.; Schuster, G. B.; Barnett, R. N.; Cleveland, C. L.; Landman, U. Oxidation of DNA: Damage to Nucleobases. Acc. Chem. Res. 2010, 43, 280-287.

(7) Genereux, J. C.; Barton, J. K. Mechanisms for DNA Charge Transport. Chem. Rev. 2010, 110, 1642-1662.

(8) Kawai, K.; Majima, T. Hole Transfer Kinetics of DNA. Acc. Chem. Res. 2013, 46, 2616-2625.

(9) Palecek, E.; Bartosik, M. Electrochemistry of Nucleic Acids. Chem. Rev. 2012, $112,3427-3481$.

(10) Pluharova, E.; Slavicek, P.; Jungwirth, P. Modeling Photoionization of Aqueous DNA and Its Components. Acc. Chem. Res. 2015, 48, 1209-1217.

(11) Kumar, A.; Adhikary, A.; Sevilla, M. D.; Close, D. M. One-electron oxidation of ds(5 '-GGG-3 ') and ds(5 '-G(8OG)G-3 ') and the nature of hole distribution: a density functional theory (DFT) study. Phys. Chem. Chem. Phys. 2020, 22, 5078-5089.

(12) Candeias, L. P.; Steenken, S. Stucture and acid-base properties of one-electronoxidized deoxyguanosine, guanosine, and 1-methylguanosine. J. Am. Chem. Soc. 1989, 111, 1094-1099.

(13) O'Neill, P.; Parker, A. W.; Plumb, M. A.; Siebbeles, L. D. A. Guanine modifications following ionization of DNA occurs predominantly via intra- and not interstrand charge migration: An experimental and theoretical study. J. Phys. Chem. B 2001, 105, 52835290.

(14) Melvin, T.; Plumb, M. A.; Botchway, S. W.; Oneill, P.; Parker, A. W. 193 nm Light Induces Single-strand Breakage of DNA Predominantly at Guanine. Photochem. Photobiol. 1995, 61, 584-591. 
(15) Marguet, S.; Markovitsi, D.; Talbot, F. One and two photon ionization of DNA single and double helices studied by laser flash photolysis at $266 \mathrm{~nm}$. J. Phys. Chem. B 2006, 110, 11037-11039.

(16) Gabelica, V.; Rosu, F.; Tabarin, T.; Kinet, C.; Antoine, R.; Broyer, M.; De Pauw, E.; Dugourd, P. Base-dependent electron photodetachment from negatively charged DNA strands upon 260-nm laser irradiation. J. Am. Chem. Soc. 2007, 129, 4706-4713.

(17) Gomez-Mendoza, M.; Banyasz, A.; Douki, T.; Markovitsi, D.; Ravanat, J. L. Direct Oxidative Damage of Naked DNA Generated upon Absorption of UV Radiation by Nucleobases. J. Phys. Chem. Lett. 2016, 7, 3945-3948.

(18) Mohammad, T.; Morrison, H. Evidence for the photosensitized formation of singlet oxygen by UVB irradiation of 2'-deoxyguanosine 5'-monophosphate. J. Am. Chem. Soc. 1996, 118, 1221-1222.

(19) Yagura, T.; Schuch, A. P.; Machado Garcia, C. C.; Reily Rocha, C. R.; Moreno, N. C.; Friedmann Angeli, J. P.; Mendes, D.; Severino, D.; Sanchez, A. B.; Di Mascio, P.; Gennari de Medeiros, M. H.; Martins Menck, C. F. Direct participation of DNA in the formation of singlet oxygen and base damage under UVA irradiation. Free Rad. Biol. Med. 2017, 108, 8693.

(20) Candeias, L. P.; Steenken, S. Ionization of purine nucleosides and nucleotides and their components by 193-nm laser photolysis in aqueous solution: model studies for oxidative damage of DNA. J. Am. Chem. Soc. 1992, 114, 699-704.

(21) Banyasz, A.; Ketola, T.; Muñoz-Losa, A.; Rishi, S.; Adhikary, A.; Sevilla, M. D.; Martinez-Fernandez, L.; Improrta, R.; Markovitsi, D. UV-induced Adenine Radicals Induced in 
DNA A-tracts: Spectral and Dynamical Characterization J. Phys. Chem. Lett. 2016, 7, 39493953.

(22) Banyasz, A.; Ketola, T.; Martinez-Fernandez, L.; Improta, R.; Markovitsi, D. Adenine radicals generated in alternating AT duplexes by direct absorption of low-energy UV radiation. Faraday Disc. 2018, 207, 181-197.

(23) Banyasz, A.; Martinez-Fernandez, L.; Improta, R.; Ketola, T. M.; Balty, C.; Markovitsi, D. Radicals generated in alternating guanine-cytosine duplexes by direct absorption of low-energy UV radiation. Phys. Chem. Chem. Phys. 2018, 20, 21381-21389.

(24) Balanikas, E.; Banyasz, A.; Baldacchino, G.; Markovitsi, D. Populations and Dynamics of Guanine Radicals in DNA strands: Direct versus Indirect Generation. Molecules 2019, 24, 2347.

(25) Schultze, P.; Hud, N. V.; Smith, F. W.; Feigon, J. The effect of sodium, potassium and ammonium ions on the conformation of the dimeric quadruplex formed by the Oxytricha nova telomere repeat oligonucleotide $\mathrm{d}(\mathrm{G}(4) \mathrm{T}(4) \mathrm{G}(4))$. Nucl. Ac. Res. 1999, 27, 3018-3028.

(26) Banyasz, A.; Martinez-Fernandez, L.; Balty, C.; Perron, M.; Douki, T.; Improta, R.; Markovitsi, D. Absorption of Low-Energy UV Radiation by Human Telomere GQuadruplexes Generates Long-Lived Guanine Radical Cations. J. Am. Chem. Soc. 2017, 139, 10561-10568.

(27) Balanikas, E.; Banyasz, A.; Baldacchino, G.; Markovitsi, D. Guanine Radicals Generated in Telomeric G-quadruplexes by Direct Absorption of Low-Energy UV Photons: Effect of Potassium Ions. Molecules 2020, 25, 2094. 
(28) Banyasz, A.; Balanikas, E.; Martinez-Fernandez, L.; Baldacchino, G.; Douki, T.; Improta, R.; Markovitsi, D. Radicals generated in tetramolecular guanine quadruplexes by photoionization: spectral and dynamical features. J. Phys. Chem. B 2019, 123, 4950-4957.

(29) Behmand, B.; Balanikas, E.; Martinez-Fernandez, L.; Improta, R.; Banyasz, A.; Baldacchino, G.; Markovitsi, D. Potassium Ions Enhance Guanine Radical Generation upon Absorption of Low-Energy Photons by G-quadruplexes and Modify Their Reactivity. J. Phys. Chem. Lett. 2020, 11, 1305-1309.

(30) Behmand, B.; Balanikas, E.; Martinez-Fernandez, L.; Improta, R.; Banyasz, A.; Baldacchino, G.; Markovitsi, D. Correction to "Potassium Ions Enhance Guanine Radical Generation upon Absorption of Low-Energy Photons by G-quadruplexes and Modify Their Reactivity". J. Phys. Chem. Lett. 2020, 11, 2742-2742.

(31) Hud, V.; Plavec, J.: The role of cations in determining quadruplex structure and stability. In Quadruplex nucleic acids; Balasubramanian, S., Neidle, S., Eds.; RSC Publishing: Cambridge, 2006; pp 100-130.

(32) von Sonntag, C.: Free-Radical-Induced DNA damage and its Repair SpringerVerlag: Berlin-Heildelberg, 2006.

(33) Torche, F.; Marignier, J. L. Direct Evaluation of the Molar Absorption Coefficient of Hydrated Electron by the Isosbestic Point Method. J. Phys. Chem. B 2016, 120, 7201-7206.

(34) Kumar, A.; Becker, D.; Adhikary, A.; Sevilla, M. D. Reaction of Electrons with DNA: Radiation Damage to Radiosensitization. Int. J.Mol. Sci. 2019, 20.

(35) Peters, J. P.; Maher, L. J., III. DNA curvature and flexibility in vitro and in vivo. Q. Rev. Bioph. 2010, 43, 23-63. 
(36) Capobianco, A.; Caruso, T.; D'Ursi, A. M.; Fusco, S.; Masi, A.; Scrima, M.; Chatgilialoglu, C.; Peluso, A. Delocalized Hole Domains in Guanine-Rich DNA Oligonucleotides. J. Phys. Chem. B 2015, 119, 5462-5466.

(37) Sun, W. M.; Varsano, D.; Di Felice, R. Effects of G-Quadruplex Topology on Electronic Transfer Integrals. Nanomaterials 2016, 6 .

(38) Martinez-Fernandez, L.; Banyasz, A.; Markovitsi, D.; Improta, I. Topology controls the electronic absorption delocalization of electron hole in guanine quadruplexes. Chem. Europ. J. 2018, 24, 15185-15189.

(39) Schiedt, J.; Weinkauf, R.; Neumark, D. M.; Schlag, E. W. Anion spectroscopy of uracil, thymine and the amino-oxo and amino-hydroxy tautomers of cytosine and their water clusters. Chem. Phys. 1998, 239, 511-524.

(40) Ida, R.; Wu, G. Direct NMR detection of alkali metal ions bound to G-quadruplex DNA. J. Am. Chem. Soc. 2008, 130, 3590-3602.

(41) Hua, Y.; Changenet-Barret, P.; Improta, R.; Vayá, I.; Gustavsson, T.; Kotlyar, A. B.; Zikich, D.; Šket, P.; Plavec, J.; Markovitsi, D. Cation Effect on the Electronic Excited States of Guanine Nanostructures Studied by Time-Resolved Fluorescence Spectroscopy. J. Phys. Chem. C 2012, 116, 14682-14689.

(42) Chen, J.; Zhang, Y.; Kohler, B. Excited states in DNA strands investigated by ultrafast laser spectroscopy. Top. Curr. Chem. 2015, 356, 39-87.

(43) Markovitsi, D. UV-induced DNA Damage: The Role of Electronic Excited States. Photochem. Photobiol. 2016, 92, 45-51.

(44) Schreier, W. J.; Gilch, P.; Zinth, W. Early Events of DNA Photodamage. Ann. Rev. Phys. Chem. 2015, 66, 497-519. 
(45) Martinez-Fernandez, L.; Changenet, P.; Banyasz, A.; Gustavsson, T.; Markovitsi, D.; Improta, I. A Comprehensive Study of Guanine Excited State Relaxation and Photoreactivity in G-Quadruplexes. J. Phys. Chem. Lett. 2019, 10, 6873-6877.

(46) Ma, C. S.; Chan, R. C.-T.; Chan, C. T.-L.; Wong, A. K.-W.; Kwok, W.-M. Realtime Monitoring Excitation Dynamics of Human Telomeric Guanine Quadruplexes: Effect of Folding Topology, Metal Cation, and Confinement by Nanocavity Water Pool. J. Phys. Chem. Lett. 2019, 10, 7577-7585.

(47) Bucher, D. B.; Pilles, B. M.; Carell, T.; Zinth, W. Charge separation and charge delocalization identified in long-living states of photoexcited DNA. Proc. Natl. Acad. Sci. USA 2014, 111, 4369-4374.

(48) Teychene, J.; Didacus-Prins, D.; Chouini-Lalanne, N.; Sartor, V.; Dejugnat, C. Formulation induces direct DNA UVA photooxidation. Part I. Role of the formulating cationic surfactant. J. Mol. Liq. 2019, 295, 111712.

(49) Sutherland, J. C.; Griffin, K. P. Absorption spectrum of DNA for wavelengths greater than $300 \mathrm{~nm}$. Radiat. Res. 1981, 86, 399-410.

(50) Banyasz, A.; Vayá, I.; Changenet-Barret, P.; Gustavsson, T.; Douki, T.; Markovitsi, D. Base-pairing enhances fluorescence and favors cyclobutane dimer formation induced upon absorption of UVA radiation by DNA. J. Am. Chem. Soc. 2011, 133, 5163-5165.

(51) Spata, V. A.; Matsika, S. Role of Excitonic Coupling and Charge-Transfer States in the Absorption and CD Spectra of Adenine-Based Oligonucleotides Investigated through QM/MM Simulations. J. Phys. Chem. A 2014, 118, 12021-12030. 
(52) Chatgilialoglu, C.; Caminal, C.; Altieri, A.; Vougioukalakis, G. C.; Mulazzani, Q. G.; Gimisis, T.; Guerra, M. Tautomerism in the guanyl radical. J. Am. Chem. Soc. 2006, 128, 13796-13805.

(53) Kumar, A.; Sevilla, M. D. Excited States of One-Electron Oxidized GuanineCytosine Base Pair Radicals: A Time Dependent Density Functional Theory Study. J. Phys. Chem. A 2019, 123, 3098-3108.

(54) Kobayashi, K.; Tagawa, S. Direct observation of guanine radical cation deprotonation in duplex DNA using pulse radiolysis. J. Am. Chem. Soc. 2003, 125, 1021310218.

(55) Wu, L. D.; Liu, K. H.; Jie, J. L.; Song, D.; Su, H. M. Direct Observation of Guanine Radical Cation Deprotonation in G-Quadruplex DNA. J. Am. Chem. Soc. 2015, 137, 259-266.

(56) Melvin, T.; Botchway, S. W.; Parker, A. W.; Oneill, P. Induction of strand breaks in single-stranded polyribonucleotides and DNA by photoionization: One electron oxidized nucleobase radicals as precursors. J. Am. Chem. Soc. 1996, 118, 10031-10036.

(57) Ravanat, J. L.; Douki, T.; Cadet, J. Direct and indirect effects of UV radiation on DNA and its components. J. Photochem. Photobiol., B: Biology 2001, 63, 88-102.

(58) Douki, T. Effect of denaturation on the photochemistry of pyrimidine bases in isolated DNA. J. Photochem. Photobiol B 2006, 82, 45-52.

(59) Daly, S.; Porrini, M.; Rosu, F.; Gabelica, V. Electronic spectroscopy of isolated DNA polyanions. Faraday Disc. 2019, 217, 361-382. 
(60) Fleming, A. M.; Burrows, C. J. G-Quadruplex Folds of the Human Telomere Sequence Alter the Site Reactivity and Reaction Pathway of Guanine Oxidation Compared to Duplex DNA. Chem. Res.Toxicol. 2013, 26, 593-607.

(61) Mergny, J. L.; Sen, D. DNA Quadruple Helices in Nanotechnology. Chem. Rev. 2019, 119, 6290-6325.

(62) Livshits, G. I.; Stern, A.; Rotem, D.; Borovok, N.; Eidelshtein, G.; Migliore, A.; Penzo, E.; Wind, S. J.; Di Felice, R.; Skourtis, S. S.; Carlos Cuevas, J.; Gurevich, L.; Kotlyar, A. B.; Porath, D. Long-range charge transport in single G-quadruplex DNA molecules. Nat. Nanotechnol. 2014, 9, 1040-1046.

(63) Sha, R.; Xiang, L.; Liu, C.; Balaeff, A.; Zhang, Y.; Zhang, P.; Li, Y.; Beratan, D. N.; Tao, N.; Seeman, N. C. Charge splitters and charge transport junctions based on guanine quadruplexes. Nat. Nanotechnol. 2018, 13, 316-321. 